\title{
Torsion of a Wandering Spleen Treated by Laparoscopic Surgery: A Case Report
}

\section{In Cho}

Department of Surgery, Soonchunhyang University Bucheon Hospital, Soonchunhyang University College of Medicine, Bucheon, Korea

\begin{abstract}
The spleen is an organ located in the upper left portion of the abdomen. Wandering spleen is defined as the location of the spleen is the shift to other parts of the abdomen rather than the left upper quadrant. Wandering spleen is a rare clinical condition and can lead to hilar torsion and subsequent infarction requiring emergency surgery. The author presents a case of torsion of a wandering spleen in a 34-year-old female presenting with abdominal pain. The patients underwent emergent laparoscopic splenectomy. She had an uncomplicated postoperative course and recovered well.
\end{abstract}

Keywords: Wandering spleen; Torsion of spleen; Laparoscopic surgery; Case report

\section{INTRODUCTION}

A wandering spleen is a rare variation, defined as the location of the spleen shifted to other parts of the abdomen rather than the left upper quadrant [1]. A normal spleen is attached to several ligaments that normally fix the spleen to the region below the left dome of the diaphragm. As a result of an absence or loosening of these splenic ligaments, the spleen could move to other parts of the abdomen and rotate by itself. This condition is very rare, with an incidence of less than $0.2 \%$ [2], and makes susceptible the spleen to acute torsion and infarction with splenic vein obstruction, leading to the formation of gastric varices [3]. Although many conservative treatment options have been reported for the treatment of wandering spleen, the most effective and safest option is accepted to be surgery [4].

Herein, we present a 34-year-old female with a wandering spleen with splenic torsion that was successfully treated by laparoscopic splenectomy.

\section{CASE REPORT}

A 34-year-old female was presented to the emergency depart- ment with a history of abdominal pain with nausea for 2 weeks. She reported 10 days of mild left upper quadrant abdominal pain, with a sudden increase in the intensity of pain for the last few days. The pain was intermittent and then turned to continuous and severe before the patient was admitted to the hospital. There was no history of fever or trauma. She underwent laparoscopic appendectomy for acute appendicitis 4 years ago. She gave birth to a child 2 months ago.

On arrival at the emergency department, the patient was afebrile $\left(36.4^{\circ} \mathrm{C}\right)$, pulse rate of 98 beats/min, blood pressure of $120 / 85$ $\mathrm{mm} \mathrm{Hg}$, respiratory rate of 20 breaths/min, and pulse oximetry of $100 \%$ on room air. She appeared in moderate distress from pain; however, no pallor, jaundice, finger clubbing, or lymphadenopathy was noticed. On abdominal physical examination, abdominal distension was noted. There was diffuse abdominal tenderness more prominent in the epigastric area, but no definite palpable mass was detected.

Laboratory parameters showed white blood cell counts of $6.83 \times$ $10^{3} / \mu \mathrm{L}$, hemoglobin level of $13.5 \mathrm{~g} / \mathrm{dL}$, platelet count of $193 \times 10^{3} / \mu \mathrm{L}$, C-reactive protein level of $0.39 \mathrm{mg} / \mathrm{L}$ (reference, $0.0-0.5 \mathrm{mg} / \mathrm{L}$ ), erythrocyte sedimentation rate of $6 \mathrm{~mm} / \mathrm{hr}$ (reference, $0-27 \mathrm{~mm} / \mathrm{hr}$ ), serum amylase level of $102 \mathrm{U} / \mathrm{L}$ (reference, 28-100 U/L), and se- 

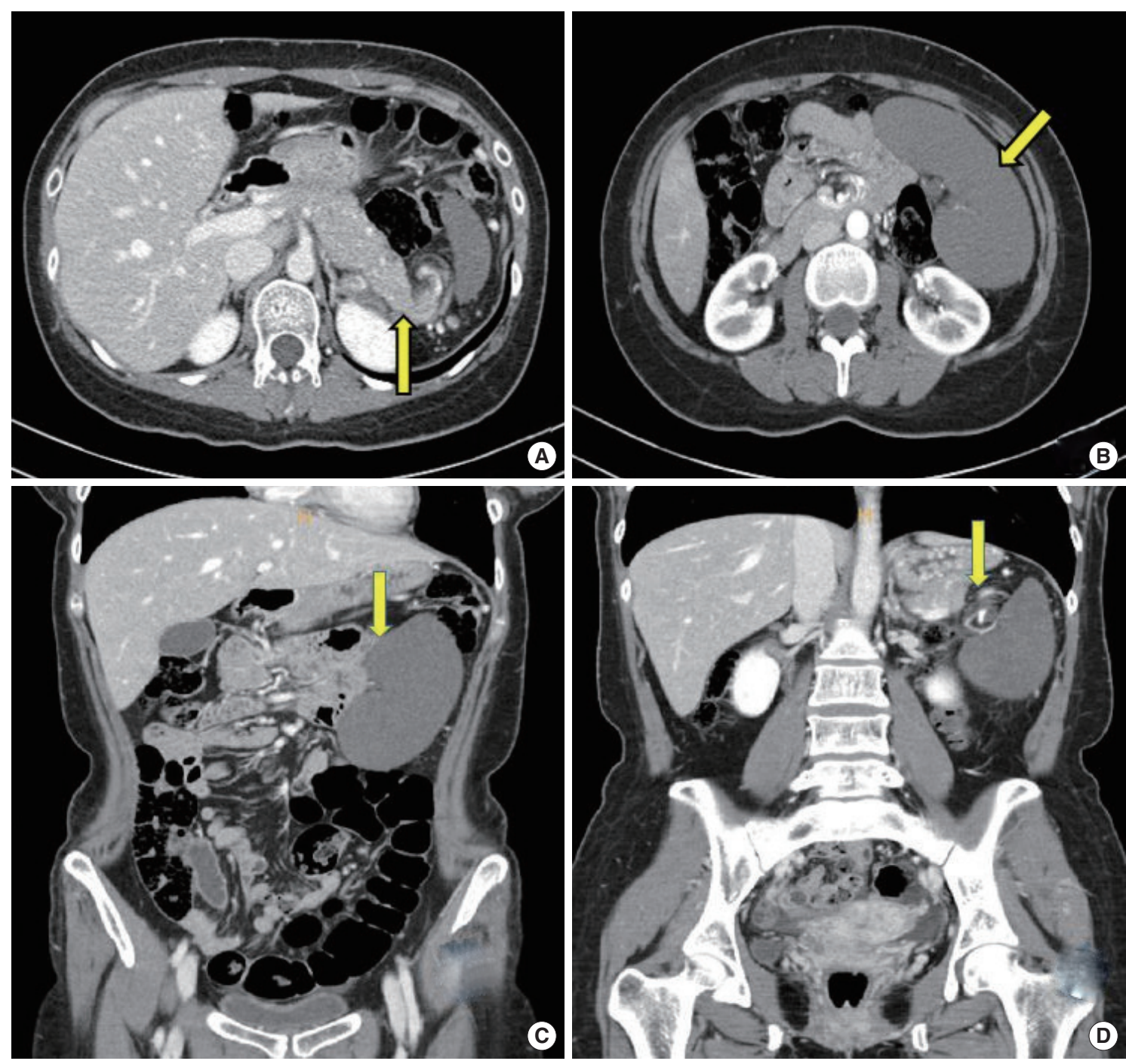

Fig. 1. Computed tomography scan showed swirling of the splenic vascular pedicle (A, white arrow) and splenomegaly and infarction of spleen (B, white arrow). Coronal image $(C, D)$

rum lipase level of $31 \mathrm{U} / \mathrm{L}$ (reference, 7-60 U/L). Liver function tests and electrolytes were normal values.

Urgent computed tomography (CT) scan of abdomen and pelvis with contrast was performed, which demonstrated findings consistent with splenomegaly and developed splenic infarction, with swirling of the splenic vascular pedicle (Fig. 1). She was taken emergently to the operating room for laparoscopic exploration with splenectomy. A total of three trocars were used in the operation. Intraoperatively, her spleen was noted as congested and twisted on the left abdomen (Fig. 2). Laparoscopic splenectomy was performed successfully, and the pathologic result of specimen was no diagnostic abnormalities. Her postoperative course was uneventful, and she was discharged on the 5th postoperative day. After 6 months of operation, she visited the outpatient clinic for routine examination. She was healthy and performed the usual activities of daily living. The incision scars 6 months after surgery had no specific features (Fig. 3).

The patient provided oral informed consent for the publication of clinical details and images.

\section{DISCUSSION}

The wandering spleen is a rare entity that was first described in 1854 by the Polish physician Jozef Doetl [5]. The spleen is normally located in the left upper quadrant of abdomen fixed by several ligaments. These ligaments are derived from the dorsal mesogastri- 

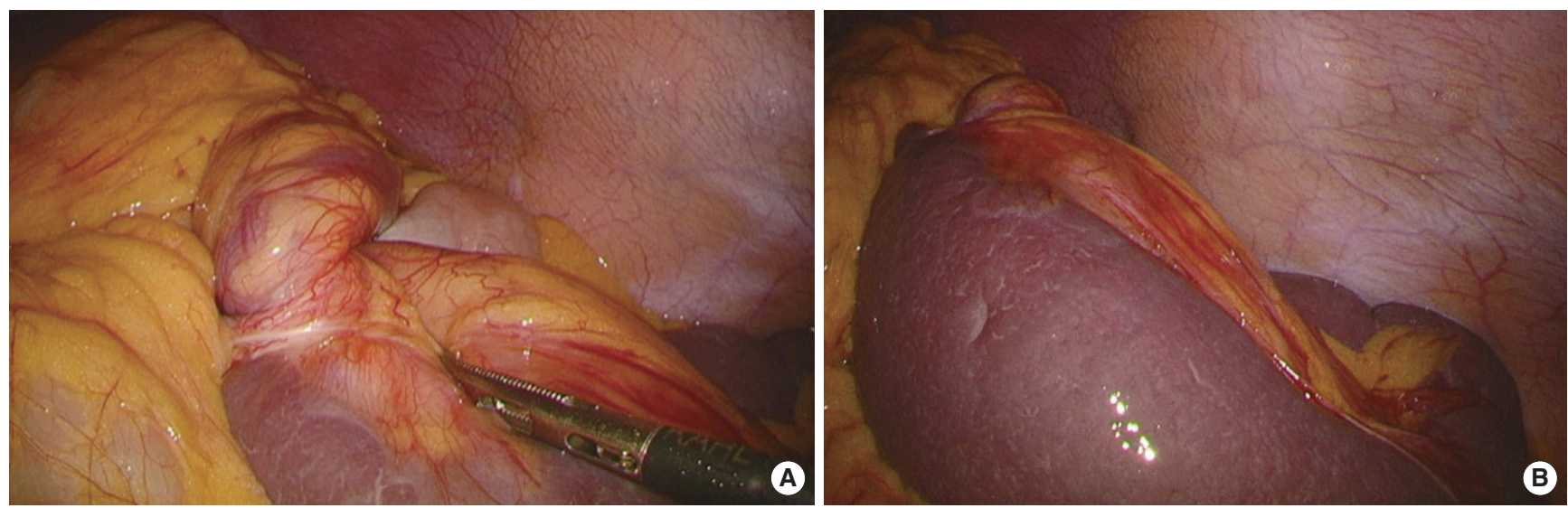

Fig. 2. Intraoperative image showing the torsion of splenic vascular pedicle and consequent several engorged veins (A) and enlarged spleen (B).

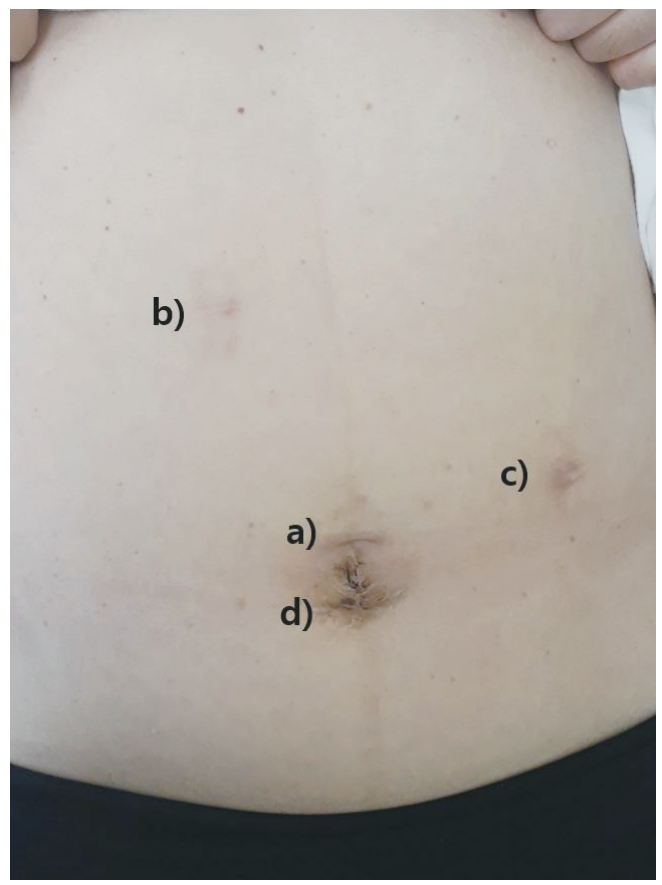

Fig. 3. Incision scars after 6 months of operation. ${ }^{\text {al } 12-m m ~ t r o c a r ~ s i t e ~ f o r ~ c a m-~}$ era, ${ }^{\text {b) } 5-m m ~ t r o c a r ~ s i t e, ~}{ }^{c / 12-m m ~ t r o c a r ~ s i t e, ~ a n d ~ d) p r e v i o u s ~ i n c i s i o n ~ s c a r ~ f o r ~ l a p a-~}$ roscopic appendectomy (4 years ago).

um. Among the ligaments, the splenorenal ligament anchors the spleen to the left kidney. The gastrosplenic ligament and splenocolic ligament hold the spleen to the posterior aspect of the stomach and left colic flexure, respectively. The phrenicosplenic ligament anchors the spleen to the left diaphragm [6]. If these ligaments have not been developed or failed to their function, the spleen wanders into the abdominal cavity.

The etiology of wandering spleen includes congenital causes of the inadequate fusion between the abdominal wall and dorsal me- sogastrium during embryogenesis and acquired causes such as trauma, ligament laxity, and pregnancy [7]. Wandering spleen by congenital causes is common in children between 3 months and 10 years of age. The acquired wandering spleen usually occurs in young women aged $20-40$ years and is 10 times more frequent in multiparous women due to laxity of the abdominal wall and hormonal changes as a result of pregnancy [8].

When diagnosing patients suspected of wandering spleen with torsion, a contrast-enhanced CT scan is considered as the modality of choice $[6,9]$. CT findings could give clinicians various information about the size and position of the spleen, the appearance of the splenic pedicle, and the viability of the spleen. This information is essential for making a decision about performing the emergency operation.

In young and asymptomatic patients, preservation of the spleen is recommended; therefore, the splenopexy is considered a reasonable surgical option for these patients [10]. On the contrary, emergency splenectomy should be performed when there is obvious evidence of splenic infarction. Currently, minimally invasive surgeries such as laparoscopic approach for the splenopexy and splenectomy were widely performed owing to the advances in laparoscopic instruments, devices, and techniques. Therefore, in the current case, splenectomy was performed because the splenic infarction was revealed on the CT scan, and laparoscopic approach was decided to take advantage of minimally invasive surgery.

The torsion of wandering spleen is rare in patients presenting with acute abdominal pain and extremely difficult to detect early. Contrast enhanced CT scan is crucial in making an accurate and timely diagnosis. When torsion and infarction of wandering spleen are diagnosed, splenectomy is the treatment of choice. Lap- 
aroscopic splenectomy is feasible and less invasive than open splenectomy. We report a rare case of wandering spleen with splenic torsion requiring the emergency operation, and that was successfully treated by laparoscopic splenectomy.

\section{CONFLICT OF INTEREST}

No potential conflict of interest relevant to this article was reported.

\section{REFERENCES}

1. Goksu M, Baykan AH. Torsion of wandering spleen: a case report. J Emerg Med 2020;58:e189-92.

2. Viana C, Cristino H, Veiga C, Leao P. Splenic torsion, a challenging diagnosis: case report and review of literature. Int J Surg Case Rep 2018;44: 212-6.
3. Chue KM, Tan JKH, Pang NQ, Kow AWC. Laparoscopic splenectomy for a wandering spleen with resultant splenomegaly and gastric varices. ANZ J Surg 2020;90:2124-5.

4. Termos S, Redha A, Zbibo R, Alduwaisan A, AlKabbani M, Elyousif N, et al. Torsion of huge wandering accessory spleen: case report and review of literature. Int J Surg Case Rep 2017;38:131-5.

5. Magowska A. Wandering spleen: a medical enigma, its natural history and rationalization. World J Surg 2013;37:545-50.

6. Reisner DC, Burgan CM. Wandering spleen: an overview. Curr Probl Diagn Radiol 2018;47:68-70.

7. Karapolat B, Korkmaz HAA, Kocak G. Torsion of the wandering spleen. Am J Med Sci 2019;357:e17-8.

8. Ben Ely A, Seguier E, Lotan G, Strauss S, Gayer G. Familial wandering spleen: a first instance. J Pediatr Surg 2008;43:E23-5.

9. Parada Blazquez MJ, Rodriguez Vargas D, Garcia Ferrer M, Tinoco Gonzalez J, Vargas Serrano B. Torsion of wandering spleen: radiological findings. Emerg Radiol 2020;27:555-60.

10. Awan M, Gallego JL, Al Hamadi A, Vinod VC. Torsion of wandering spleen treated by laparoscopic splenopexy: a case report. Int J Surg Case Rep 2019;62:58-61. 